\title{
The role of foot collateral vessels on angiosome-oriented revascularization
}

\author{
César Varela, Francisco Acín, Joaquin De Haro, Ignacio Michel \\ Department of Angiology and Vascular Surgery, Hospital Universitario de Getafe, Getafe, Madrid, Spain \\ Correspondence to: César Varela. Department of Angiology and Vascular Surgery, Hospital Universitario de Getafe, Ctra. Toledo, Km 12.5, 28905 Madrid, \\ Spain. Email: cvarelacasariego@gmail.com. \\ Provenance: This is an invited Editorial commissioned by Section Editor Dr. Kaiping Zhang, PhD (AME College, AME Group, China). \\ Comment on: Špillerová K, Settembre N, Biancari F, et al. Angiosome Targeted PTA is More Important in Endovascular Revascularisation than in \\ Surgical Revascularisation: Analysis of 545 Patients with Ischaemic Tissue Lesions. Eur J Vasc Endovasc Surg 2017;53:567-75.
}

Submitted Aug 14, 2017. Accepted for publication Aug 21, 2017.

doi: $10.21037 / \mathrm{atm} .2017 .08 .41$

View this article at: http://dx.doi.org/10.21037/atm.2017.08.41

For many years, it has been generally accepted that distal vein bypass revascularization performed to the less diseased angiographic tibial vessel was the most effective treatment for critical limb ischemia (CLI) and tissue loss, as long as a direct line of blood flow to the foot was obtained. The main objective of the revascularization procedure is to obtain sufficient foot perfusion to ensure ischemic ulcer healing, since delayed healing increases the risk of limb loss (1-3). However, the meteoric endovascular therapy technology developments seen in the last decades have radically changed this paradigm. Nowadays, distal vein bypass still stands as the gold standard treatment for CLI revascularization due to its greater durability and its proven effectiveness. Nevertheless, endovascular tibial procedures represent an attractive option for CLI patients, usually frail elderly individuals with diabetes mellitus and severe comorbidities, because of its lower procedure-related complications when compared with bypass. Furthermore, according to several studies, tibial endovascular procedures could provide similar clinical outcomes as open surgical reconstruction surgery $(4,5)$. For these reasons, many vascular surgeons have implemented the endovascular therapy as first line of treatment for CLI.

This change of view, coupled with the fact that up to $15 \%$ of technically successful infrainguinal revascularization procedures performed for CLI are associated with ulcer healing failure and limb loss (6), has led to an increased interest in the clinical effect of reperfusion depending on the different anatomical areas of the foot. In other words, ulcers may fail to heal because of inadequate vascular connections between the revascularized tibial artery and the local ischemic area. Thus, obtaining a direct line of blood flow to the foot may not be enough to optimize pedal wound healing.

As this theory has gained popularity, many centres apply an angiosome-oriented revascularization for CLI revascularization planning. The anatomical concept of angiosomes is widely used in modern plastic surgery. As first described by Taylor and Palmer, an angiosome is a 3-dimensional block of tissue supplied by a specific source artery and drained by a specific vein (7). The foot can be divided into 6 angiosomes arising from the posterior tibial artery $(\mathrm{n}=3)$, the anterior tibial artery $(\mathrm{n}=1)$ and the peroneal artery $(\mathrm{n}=2$, Figure 1). Each angiosome is interconnected by a vast "small sized" collateral network called chokevessel system. These anatomical structures are also linked by "medium sized vessels" (arterial-arterial connections) that arise from distal tibial vessels and its branches, which allow the development of alternative routes of blood flow if the direct route is compromised $(8,9)$. The most important arterial-arterial connections of the foot are the plantar arch and the distal peroneal branches.

Direct revascularization (DR) of the injured angiosome through its specific tibial artery has shown improved results in terms of healing and limb salvage in multiple retrospective cohort studies and meta-analysis (3,10-13). In fact, the latest AHA/ACC guideline on the management of patients with lower extremity peripheral artery disease suggests that an angiosome-directed endovascular therapy may be reasonable for patients with CLI and nonhealing wounds or gangrene (14). 

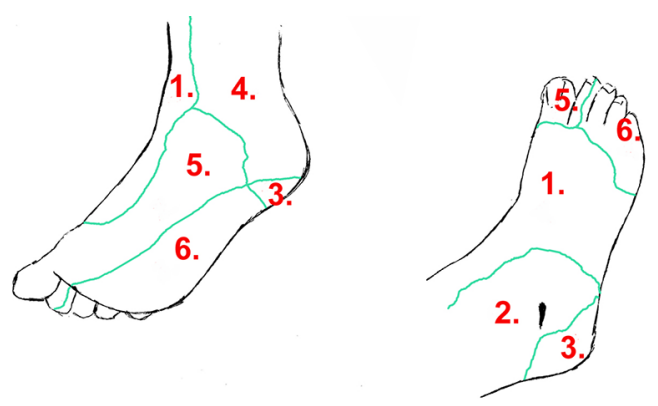

Figure 1 Angiosomes of the foot. Dorsalis pedis artery [1] of the anterior tibial artery. Anterior branch [2] and calcaneal branch [3] of the peroneal artery. Calcaneal branch [4], medial plantar branch [5] and lateral plantar branch [6] of the posterior tibial artery.

In this context, a few months ago, Špillerová et al. published an interesting retrospective observational study performed in 545 diabetic patients with tissue loss (15). The relevance of their research is that it is the only one to date that compares surgical versus endovascular revascularization using the angiosome concept in diabetic patients with ischemic ulcers. The authors observed that in diabetics, indirect revascularization (IR) of the injured angiosome through a non-specific tibial artery using endovascular procedures leads to significant worse healing and limb salvage rates compared with DR. However, in the bypass arena, achieving a DR or IR offered similar results. Woundhealing rates were better after bypass surgery than after endovascular procedures, independent of the angiosome orientation. The authors conclude that endovascular procedures should be targeted according to the angiosome concept. However, in bypass procedures the artery with best runoff should be selected as the outflow artery. These results are complementary to those published by the same group in a previous large cohort of revascularizated patients that included $60 \%$ of diabetics (16).

Unfortunately, studies published by the Špillerová group, as most of the published researches on the topic, present several design limitations that may prevent the applicability of its results. Most studies are retrospective with all the drawbacks that this entails. Ischemic ulcers are not described in detail. Ulcer topical treatment, as well as the need for ulcer surgical debridement or minor amputations, is frequently not characterized. Patient's vascular anatomy prior to revascularization, specifically foot distal vessels, is usually not analyzed. Furthermore, the revascularization procedure characteristics are not described in depth in most of the studies. As Špillerová et al. admit, without doubt, well-planned prospective studies are needed to confirm the usefulness of the angiosome concept in the treatment of CLI.

Having this aim in mind, it is necessary to take into account the whole set of factors that influence the ischemic ulcer healing process. Such factors can be grouped into three areas: (I) patient-dependent factors, like hypoalbuminemia, diabetes mellitus and chronic renal disease which could affect negatively the healing process independently of the results of revascularization $(5,15,17)$; (II) procedure-related factors, where it is necessary to study in greater depth the hemodynamic remodelling that occurs in a critically ischemic limb after an endovascular or surgical reperfusion and the influence of these hemodynamic changes on collateral foot vessels and peripheral vascular bed function. And lastly, (III) ulcer-dependent factors, like the location of the wound on the foot, as well as the extension, depth and presence of infection (18).

It is widely accepted that infrainguinal vein bypass patency depends on a good runoff quality. Achieving a direct line of blood flow to the foot is sufficient to preserve the limb and bypass thrombosis often requires reinterventions with high risk of future limb loss $(1,2)$. Therefore, as concluded by Špillerová's study (15), we do not support that an angiosome-oriented strategy has, nowadays, a clear clinical utility in the field of open bypass surgery. However, such strategy may by be useful in endovascular revascularization planning, since this type of therapy allows the treatment of more than one tibial vessel, including vessels below the ankle $(3,19)$. Therefore, in our opinion, the interest of Špillerová et al. research is more pathophysiological than clinical, because it raises several enthralling unanswered questions on revascularization physiology that need to be clarified by future studies. For instance, what might be the underlying mechanism for different results between surgical and endovascular revascularization according to an angiosome model of reperfusion? Or which are the mechanisms that justify different revascularization outcomes in diabetic patients from the perspective of the angiosome concept?

In order to answer these questions, we must identify several important disadvantages of the angiosome concept when it is applied to CLI with tissue loss, which has led to multiple definitions that makes comparisons between studies challenging. Angiosomes were designed as dynamic concept to be applied to non-vascular patients. In a healthy subject, the choke vessel network represents a notable rescue system when an angiosome becomes ischemic, leading to choke vessel-related collateral development in 4 to 
10 days. However, the patient with CLI chronically develops skin microcirculatory impairment related to endothelial dysfunction, altered hemorheology and white blood cell abnormal activation. Furthermore, if diabetes mellitus coexists, microcirculatory impairment worsens due to diabetic functional microangiopathy, lower limb neuropathy and foot local sepsis (19). This situation could lead to foot choke collateral network dysfunction in CLI patients, especially in diabetic ones. In addition, several anatomical variations that can influence the angiosome anatomical division of the foot have been identified (19). Moreover, the foot vascular anatomy is seriously affected in CLI, specially in diabetics and in patients with end-stage renal disease, with a high prevalence of dorsalis pedis, plantar arteries, plantar arch or distal peroneal branches occlusions. In this situation the regional vascular foot perfusion cannot be statically schematized. In fact, diffuse distal vascular lesions prevent the DR of the injured angiosome in more than a half of the revascularization procedures. Patent foot medium-sized collateral vessels (arterial-arterial connections) could be the only way to achieve ulcer local blood perfusion (19). That is why we suggest that we should seek a foot collateral-vessel guided revascularization for at least a half of distal endovascular procedures in which an IR is obtained.

Surprisingly, few studies have investigated the role of foot collateral vessels in revascularization outcomes. In the cited article by Špillerová et al., patient's angiographies of the IR group were classified as "good" or "non-existent collaterals" and the authors found that foot collateral vessels patency did not influence bypass clinical results. In the endovascular treatment group, IR patients presented a tendency towards better healing rates and significant limb salvage improvements when foot collateral vessels patency was identified (15). However, there was no data about the anatomical relationship between these collateral vessels and the injured ischemic area of the foot. In our experience, the restoration of blood flow to the ulcer through medium-sized collateral vessels (plantar arch or distal peroneal branches) provided similar clinical results in terms of ulcer healing and limb salvage to those obtained through its specific source artery $(3,11,19)$. Our outcomes are consistent with those observed by other groups and have been included in several meta-analysis (13).

From a hemodynamic perspective, an arterial occlusion represents a complex hemodynamic circuit that includes an injured artery that develops a parallel system of preexisting collateral arteries. This system connects the inflow to a distal vascular bed and enlarges when the limb becomes ischemic. Acute changes in collateral resistance in response to hyperaemic stimulus are relative small. Revascularization procedures overcome this resistance, however this process could be different depending on the type of revascularization (20). A good quality infrainguinal vein bypass is able to carry a large amount of blood flow with low-pressure gradients. Nevertheless, endoluminal therapy drives blood flow through diffused injured arteries. The diameter of an endovascular-treated tibial artery could be lower than a bypass conduit and is subject to early restenosis. Therefore, the quantity of blood flow obtained after an endoluminal procedure could be lower when compared to bypass surgery. Distal blood flow achieved through any type of vascular reconstruction must deal with other arterial occlusions, which have usually established another collateral network system on the foot. Bypass procedures could be less-dependent on foot collateral vessels patency because this type of revascularization drives a greater amount of blood flow. In this setting, collateral network hemodynamic properties could be completely maximized even in the case of poor quality distal vessels. On the other hand, the lesser amount of flow obtained after an endovascular revascularization may require better foot collateral vessels quality in order to drive enough oxygen to the injured peripheral vascular bed. This hemodynamic reasoning may partially explain why in Špillerová research and in others studies IR of the ischemic ulcer offered worse clinical outcomes in endovascular treatment than in bypass surgery $(13,15)$. In many of these studies, foot collateral network was not analyzed in the IR group. Špillerová et al. found improved outcomes when "good collaterals" were identified in the IR endovascular group, which also support our argument (15). Furthermore, as previously commented, diabetes mellitus in CLI patients is associated with choke vessel network depletion and with occlusion of distal medium-sized foot vessels (19). Therefore, revascularization procedures in diabetic patients could be more dependent on foot collateral vessels quality (21). Moreover, IR could be more frequent in diabetic patients because peroneal artery that connects to the foot through its distal collateral branches is relative spared from terminal stages of atherosclerosis and it is one of the last tibial vessels that becomes occluded in these patients $(3,11,19)$. On the other hand, in Špillerová's studies, bypass procedures yielded better ulcer-healing rates when compared to endovascular procedures $(15,16)$. These results are consistent with those observed in other researches (13). We also found a trend 
toward a faster healing time after bypass surgery than after endovascular procedures, although our results were not statistically significant (5). The greater amount of blood flow obtained with bypass procedures may justify these findings.

Foot collateral vessels patency could also be crucial in several situations that require higher ulcer metabolic activity or in case of a severe peripheral vascular bed dysfunction, especially if DR of the injured angiosome cannot be achieved. Diabetic microangiopathy induces by itself a reduction in skin tissue oxygenation (19), which may be partially counteracted by a greater oxygen supply if capillary blood flow is increased through collateral vessels. On the other hand, the ischemic wound requires more energy input to overcome infection. Thus, good quality arterial connections between the revascularized tibial artery and the infected ulcer may be needed. In Špillerová research and in other studies, high C-reactive protein levels (CRP) were associated with worse infrainguinal revascularization clinical results $(15,16,21)$. Infections are more severe in diabetic patients and seriously increase the risk of limb loss even after a successful revascularization procedure $(3,5,19)$. Several studies on distal revascularization have defended that the relation of CRP levels with worse clinical outcomes could be explained by ulcer infection (21). However, high CRP levels may also indicate a more advanced and diffuse vascular disease since this inflammation marker has been associated with atherosclerosis extension. In addition, the chronic inflammation process that surrounds atherosclerosis is closely linked with endothelial dysfunction and may negatively influence the results of revascularization through peripheral vascular bed functional impairment (22).

In recent years, several authors have performed small translational studies that measured the foot microcirculation function in revascularizated patients with CLI. Microcirculation function was analyzed by means of quantitative imaging test (such as perfusion angiography or indocyanine green angiography) or other betterknown diagnostic tools (such as skin perfusion pressure or transcutaneous oxygen pressure tests) (23-25). Most of these studies were designed to validate these tests in the clinical setting and their follow-up periods were usually short. It is worth noting that, as compared with the results of cohort studies, the outcomes of some of these researches do not support an angiosome-oriented strategy of revascularization. These differences highlight the need of a better knowledge of limb reperfusion physiology process after distal revascularization procedures. In these regard, we deem that future prospective well-designed studies- which include large cohorts and consider the three areas that influence the ulcer healing process, together with the anatomic, hemodynamic and functional aspects of revascularization-will surely improve clinical outcomes and the quality of life of this frail group of patients. As we await these studies, we suggest a distal endovascular planning in three steps. First, it is mandatory to obtain a direct straight line to the foot through the easier-to-treat tibial artery, even if the injured angiosome is not anatomically fed. Second, if in the first step we did not achieve blood flow to the ulcer it is advisable to attempt the revascularization of another tibial vessel specifically related to the ulcer or indirectly related trough collateral vessels. Third, if it is not possible to obtain a direct line to the foot, a distal vein bypass has to be considered if feasible.

\section{Acknowledgements}

None.

\section{Footnote}

Conflicts of Interest: The authors have no conflicts of interest to declare.

\section{References}

1. Norgren L, Hiatt WR, Dormandy JA, et al. TransAtlantic Inter-Society Concensus (TASC II). Management of peripheral arterial disease (PAD). Eur J Vasc Endovasc Surg 2007;33 Suppl 1:S1-S75.

2. Hirsch AT, Haskal ZJ, Hertzer NR, et al. ACC/ AHA 2005 practice guidelines for the management of patients with peripheral arterial disease. Circulation 2006;113:e463-e654.

3. Acin F, Varela C, López de Maturana I, et al. Results of infrapopliteal endovascular procedures performed in diabetic patients with critical limb ischemia and tissue loss from the perspective of an angiosomeoriented revascularization strategy. Int J Vasc Med 2014;2014:270539.

4. Romiti M, Albers M, Brochado-Neto FC, et al. Metaanalysis of infrapopliteal angioplasty for chronic critical limb ischemia. J Vasc Surg 2008;47:975-81.

5. Varela C, Acin F, De Haro J, et al. Influence of surgical or endovascular distal revascularization of the lower limbs on ischemic ulcer healing. J Cardiovasc Surg (Torino) 2011;52:381-9. 
6. Wölfle KD, Bruijnen H, Loeprecht H, et al. Graft patency and clinical outcome of femorodistal arterial reconstruction in diabetic and non-diabetic patients: results of a multicentre comparative analysis. Eur J Vasc Endovasc Surg 2003;25:229-34.

7. Taylor GI, Palmer JH. The vascular territories (angiosomes) of the body: experimental study and clinical applications. Br J Plast Surg 1987;40:113-41.

8. Taylor GI, Pan WR. Angiosomes of the leg: anatomic study and clinical implications. Plast Reconstr Surg 1998;102:599-616.

9. Attinger CE, Evans KK, Bulan E, et al. Angiosomes of the foot and ankle and clinical implications for limb salvage: reconstruction, incisions, and revascularization. Plast Reconstr Surg 2006;117:261S-293S.

10. Alexandrescu VA, Hubermont G, Philips Y, et al. Selective primary angioplasty following an angiosome model of reperfusion in the treatment of wagner 1-4 diabetic foot lesions: practice in a multidisciplinary diabetic limb service. J Endovasc Ther 2008; 15:580-93.

11. Varela C, Acin F, de Haro J, et al. The role of foot collateral vessels on ulcer healing and limb salvage after successful endovascular and surgical distal procedures according to an angiosome model. Vasc Endovascular Surg 2010;44:654-60.

12. Söderström M, Albäck A, Biancari F, et al. Angiosometargeted infrapopliteal endovascular revascularization for treatment of diabetic foot ulcers. J Vasc Surg 2013;57:427-35.

13. Jongsma H, Bekken JA, Akkersdijk GP, et al. Angiosomedirected revascularization in patients with critical limb ischemia. J Vasc Surg 2017;65:1208-19.e1.

14. Gerhard-Herman MD, Gornik HL, Barrett C, et al. 2016 AHA/ACC guideline on the management of patients with lower extremity peripheral artery disease: Executive summary: A report of the American College of Cardiology/ American Heart Association Task Force on clinical practice guidelines. Circulation 2017;135:e686-725.

15. Špillerová K, Settembre N, Biancari F, et al. Angiosome Targeted PTA is More Important in Endovascular Revascularisation than in Surgical Revascularisation: Analysis of 545 Patients with Ischaemic Tissue Lesions.

Cite this article as: Varela C, Acín F, De Haro J, Michel I. The role of foot collateral vessels on angiosome-oriented revascularization. Ann Transl Med 2017;5(21):431. doi: 10.21037/atm.2017.08.41
Eur J Vasc Endovasc Surg 2017;53:567-75.

16. Spillerova K, Biancari F, Leppäniemi A, et al. Differential impact of bypass surgery and angioplasty on angiosometargeted infrapopliteal revascularization. Eur J Vasc Endovasc Surg 2015;49:412-9.

17. Shiraki T, Iida O, Takahara M, et al. Predictors of delayed wound healing after endovascular therapy of isolated infrapopliteal lesions underlying critical limb ischemia patients with high prevalence of diabetes mellitus and hemodialysis. Eur J Vasc Endovasc Surg 2015;49:565-73.

18. Söderström M, Aho PS, Lepäntalo $M$, et al. The influence of the characteristics of ischemic tissue lesions on ulcer healing time after infrainguinal bypass for critical leg ischemia. J Vasc Surg 2009;49:932-7.

19. Varela C, Acin F. The role of individual collateral reserve in tissue healing for angiosome-oriented revascularization. In: Alexandrescu VA. editor. Angiosomes applications in critical limb ischemia. In search for relevance. Edizioni Minerva Medica S.p.A, 2013:39-50.

20. Zierler RE, Sumner DS. Chapter 10: Arterial physiology. In: Cronenwett JL, Johnston KW. editors. Rutherford's Vascular Surgery Eight edition. Philadelphia: Elsevier Saunders, 2014:132-49.

21. Iida O, Takahara M, Soga Y, et al. Worse limb prognosis for indirect versus direct endovascular revascularization only in patients with critical limb ischemia complicated with wound infection and diabetes mellitus. Eur J Vasc Endovasc Surg 2013;46:575-82.

22. Varela C, De Haro J, Bleda S, et al. Circulating anti-beta2glycoprotein antibodies are associated with endothelial dysfunction, inflammation, and high nitrite plasma levels in patients with intermittent claudication. Int J Inflam 2013;2013:268079.

23. Cooper KJ, Peña C, Benenati J. Determining end points for critical limb ischemia interventions. Tech Vasc Interv Radiol 2016;19:104-12.

24. Rother U, Lang W, Horch RE, et al. Microcirculation evaluated by intraoperative fluorescence angiography after tibial bypass surgery. Ann Vasc Surg 2017;40:190-7.

25. Osawa S, Terashi H, Tsuji Y, et al. Importance of the six angiosomes concept through arterial-arterial connections in CLI. Int Angiol 2013;32:375-85. 